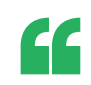

\section{translation \\ initiation \\ complexes}

are 'pushed',

not 'pulled',

by elFs in the

$5^{\prime} \rightarrow 3^{\prime}$ direction

during

scanning

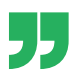

\title{
Profiling ribosome dynamics
}

Translation initiation includes the association of eukaryotic initiation factors (eIFs) and the initiator tRNA with the 40S ribosomal small subunit (SSU), which scan the $5^{\prime}$ untranslated region (5' UTR) for the initiation codon. Our understanding of translation dynamics in vivo is lacking; to address this, Archer, Shirokikh et al. developed a method to profile ribosome-mRNA interactions in live cells. The application of the method in budding yeast offered answers to several, long-standing questions regarding translation and in particular translation initiation.

The method, termed translation complex profile sequencing (TCP-seq), involves crosslinking

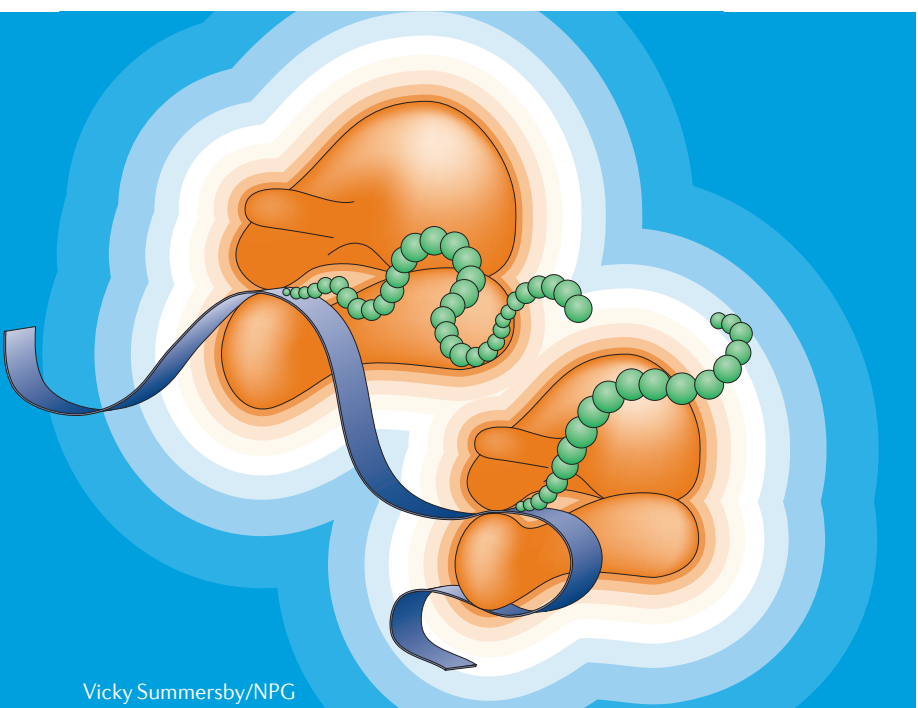

translation complexes with mRNAs in snap-chilled cells to capture the native positions of the complexes. This is followed by RNA digestion to generate ribosome-protected mRNA fragments (footprints) and separation of translation complex intermediates by sedimentation velocity. Finally, RNA is retrieved and subjected to high-throughput sequencing.

TCP-seq revealed that SSU footprints were enriched in $5^{\prime}$ UTRs, particularly at translation start codons, and near stop codons. Footprint density did not increase near the $5^{\prime}$ end of mRNAs even in longer 5' UTRs, which harboured more SSUs on average. This supports the notion that SSUs are untethered to the cap during scanning and vacate the cap proximal regions at a similar rate as they move along $5^{\prime}$ UTRs, and that multiple SSUs simultaneously scan a $5^{\prime}$ UTR.

The sizes of $5^{\prime}$ UTR SSU footprints varied and were larger than would be expected from being protected by the SSU alone. At start codons, these footprints mapped to the same 3 '-end positions, but the 5 '-end density for larger footprints declined from $\sim 40 \mathrm{nt}$ upstream of the start codons. This indicates that the extended footprint length is brought about by additional factors (probably eIFs) binding to the mRNA, but not downstream of the SSU, and implies that translation initiation complexes are 'pushed', not 'pulled', by eIFs in the $5^{\prime} \rightarrow 3^{\prime}$ direction during scanning.

The authors further analysed SSU footprints in individual mRNAs and noticed that, whereas the distribution of SSUs across many seemingly unstructured and relatively short 5' UTRs was uniform, SSU footprints at longer $5^{\prime}$ UTRs clustered at certain positions, providing evidence of scanning blockage. Blockage could be attributed to the presence of short, upstream open reading frames (ORFs), the formation of RNA structures, the presence of regulatory elements or potentially to binding by specific proteins. On the other side of the ORF, enrichment of SSU footprints of various lengths and positions was identified around the last sense codon, which indicated that frequently it is ribosomal subunits and not the mRNA that first dissociate from the translation complex upon translation termination.

TCP-seq could be used to study the function of specific translation factors as well as translation dynamics and regulation in different cellular contexts, for example, during various stress responses.

Eytan Zlotorynski

ORIGINAL ARTICLE Archer, S. K.,

Shirokikh, N. E. et al. Dynamics of ribosome

scanning and recycling revealed by translation complex profiling. Nature 535, 570-574 (2016) 